Pre-publication print, June 2016.

Bucher, Taina, and Anne Helmond. 2017. "The Affordances of Social Media Platforms." In The SAGE Handbook of Social Media, edited by Jean Burgess, Thomas Poell, and Alice Marwick. London and New York: SAGE Publications Ltd.

\title{
The Affordances of Social Media Platforms
}

Taina Bucher and Anne Helmond

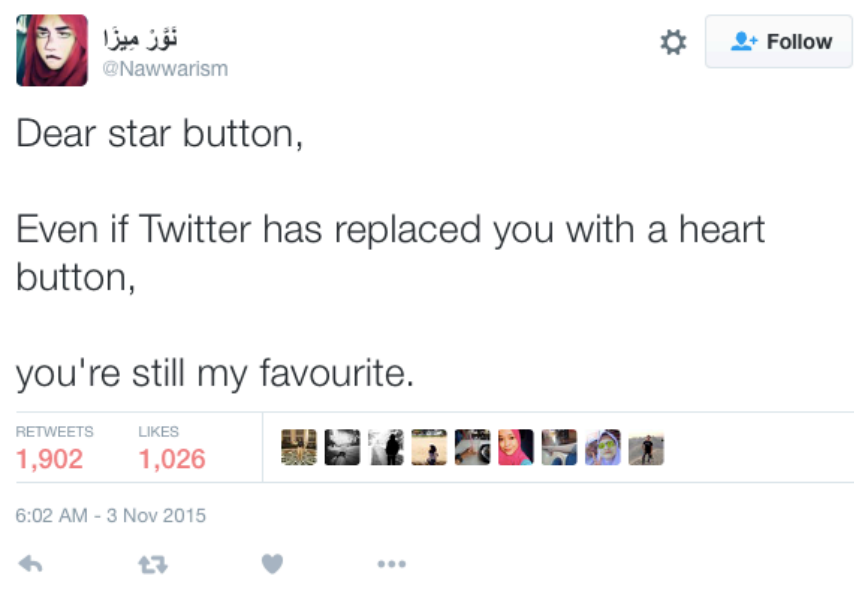

In November 2015, social media platform Twitter changed the symbol of one of its core features-the 'favorite' button-to the great disbelief of many long-time users of the platform. The favorite button, symbolized by a star and once a main feature and part of Twitter's brand identity, was changed into a much more generic like button, indicated with a heart symbol. Described by the press as 'one of the biggest changes to its platform yet' (Clifton, 2015), the Twitter change caused a storm of reactions from its end-users:

'I hate the twitter heart so hard' — (@twocitylife, Nov 3, 2015)',

'Twitter's acting like they invented the heart' - (@kumailn, Nov 3, 2015),2 
'Wait! What? When did they replace the favorite star with a heart? I'm now too scared to favorite in case somebody gets the wrong idea' — (@ForresterRobert, Nov 3, 2015) 'I'm actually sad to see the "heart" feature on the mobile app. I'd hate to see Twitter start to lose its essence. I hope this is temporary'-(@deray, Nov 3, 2015),4 "Twitter changing the "favorite" star to a "like" heart is everything that is wrong with capitalism, in icon form'-(@room34, Nov 3, 2015). ${ }^{5}$

Even Twitter engineers themselves seemed to be in disarray: ‘I work at @ twitter but even I can't believe how we replaced a completely value-neutral term like "favorite" with something so loaded' (@peterseibel, Nov 3, 2015). ${ }^{6}$ A feature is clearly not just a feature. The symbols and the connotations they carry matter. Pressing a button means something; it mediates and communicates, or as we will focus in this chapter, relates to different affordances. While this platform change may seem trivial-a controversy in the heat of the moment-it also shows how features are objects of intense feelings. Features are 'communicational actors' in the sense that they 'produce meanings and meaningfulness' (Langlois, 2014: 52). That is, as softwareproduced visual elements on an interface, features such as the favorite and like button say and suggest things. What exactly these features suggest-or afford-is not set once and for all. Clearly these features suggest the action of clicking the button, but they also assign a variety of other possibilities and interpretations. As the user reactions exemplify, Twitter buttons are endowed with different meanings, feelings, imaginings and expectations. There is the official, corporate, attempt at stabilizing the meanings, 'the heart is more expressive, enabling you to convey a range of emotions and easily connect with people' (Kumar, 2015). According to this logic, the favorite does not allow for the same kind of expressiveness. However, many users seemed to disagree. As one user suggests, 'A fav means more than a like'. ${ }^{7}$ For him the change only 'shows the homoginization [sic] of the Internet. Everything has to be LIKES' (Ibid.). Another user sees it in this way: 'Fav never meant favourite anyway. It means "I agree.", "That's funny, but not enough to RT.", or "Let's end the conversation here." ${ }^{8}$ Contrary to Twitter's official statements, many users saw the favorite as a more versatile feature than the somewhat generic 
concept of like. For others, it was precisely the fact that the favorite button wasn't a like that made the difference: 'Fav doesnt mean love/like. Fav means I want to look at the tweets after some time'. ${ }^{9}$ So if users debunked the idea of a heart button allowing a greater range of expressiveness, why change the feature in the first place?

Ultimately the change was presented by the company to provide a clearer understanding of the function to new users: 'We want to make Twitter easier and more rewarding to use, and we know that at times the star could be confusing, especially to newcomers' (Kumar, 2015). By changing the name and icon of a feature linked to a core platform activity ('favoriting'), Twitter not only standardized a mode of engagement across its services ('liking'), but also affected the perceived range of possible actions linked to these features of the platform, or its affordances.

In this chapter we want to reflect on the concept of affordance as a key term for understanding and analysing social media interfaces and the relations between technology and its users. The concept of affordance is multivalent. Originally developed in the field of ecological psychology (Gibson, 2015) and later adopted in design studies (Norman, 1988), the concept of affordance is generally used to describe what material artifacts such as media technologies allow people to do. In outlining its specific intellectual trajectory from psychology, to technology and design studies, sociology, and communication and media studies, our intention is to focus on some of the many-and sometimes conflicting-ways in which affordance has been conceptualized and operationalized across various disciplinary boundaries. Even within the field of media and communication studies there is no single way that scholars have come to understand the concept of affordance. Following the renewed debates over affordances in recent scholarship on social media (see Ilten, 2015; McVeigh-Schultz and Baym, 2015; Nagy and Neff, 2015), this chapter addresses some of the new directions in which media and communication scholars have proposed to define and analytically deploy the concept. We first describe five different-but related-ways in which affordance has been conceptualized and subsequently address how it has 
been employed to analyse social media in particular. We then outline a platform-sensitive approach to affordance as an analytical tool for examining social media based on recent examples of changes to the Twitter platform.

\section{Conceptualizing affordances}

\section{Affordances as a relational property}

The concept of affordance was originally conceived in ecological psychology by James Gibson to designate all kinds of action possibilities latent in the physical environment. Gibson first used affordance to refer to a specific kind of relationship between an animal and the environment. For Gibson, 'the affordances of the environment are what it offers the animal, what it provides or furnishes, either for good or will' (2015: 119 emphasis in original). Although this definition seems to privilege what specific environmental properties have to offer an animal, Gibson also emphasizes that affordances are to be understood as a relational property: 'I mean by it something that refers to both the environment and the animal in a way that no existing term does' (2015: 119). Gibson's aim was to propose a theory of visual perception based on the ways in which different species inhabit their surroundings. In his seminal book The Ecological Approach to Visual Perception, first published in 1979, Gibson takes up the question of 'how we see.' Gibson was interested in exploring how 'we see the environment around us', particularly in terms of how 'we see its surfaces, their layout, and their colors and textures' (2015: xiii).

His key insight was that we do not perceive the environment as such, but rather perceive it through its affordances, the possibilities for action it may provide. Fire, for example, affords warmth, illumination, and cooking, but at the same time it may also afford injury to the skin. What delineates warmth from injury is not always clear and depends on the species ability to 
detect the limit. What fire affords, then, is not merely a question of its physical properties, but its relation to a specific organism. As Gibson suggests, affordances 'have to be measured relative to the animal' (2015: 120). This does not mean, however, that affordances are indefinite. For Gibson affordances are invariant, meaning that they do "not change as the need of the observer changes. The observer may or may not perceive or attend to the affordance, according to his needs, but the affordance, being invariant, is always there to be perceived' (2015: 130 emphasis in original). In its original meaning, an affordance is conceived of as a relational property, one that 'points both ways, to the environment and to the observer' (Gibson, 2015: 121). The concept of affordance does not imply we can do anything with anything as 'different layouts afford different behaviors for different animals [...] Knee-high for a child is not the same as knee-high for an adult, so the affordance is relative to the size of the individual' (2015: 120). As Gibson put it, 'affordances do not cause behavior but constrain and control it' (1982: 411).

\section{Perceived affordances}

While Gibson's ideas have been hugely influential in a variety of fields, the concept of affordance was most notably adapted in design studies and the field of Human-Computer Interaction (HCI) through the writings of Donald Norman. First introduced in his book The Psychology of Everyday Things (1988) — which was later published as The Design of Everyday Things (1990) — Norman defined affordances as 'the perceived and actual properties of the thing, primarily those fundamental properties that determine just how the thing could possibly be used' (1988: 9). Writing in the specific context of cognitive science and design, Norman's aim was to explore the relationship between human cognition and the design of devices and everyday things. As a cognitive scientist, Norman was interested in how the human mind works, especially in terms of human errors, wanting to teach people how to avoid making mistakes. 
Norman proposes the concept of 'perceived affordance' (1990: 9, 1999a: 38) to suggest that designers can and should 'indicate how the user is to interact with the device' (1990: 8). As Norman contends, 'affordances provide strong clues to the operation of things [...] Knobs are for turning [...] Balls are for throwing or bouncing. When affordances are taken advantage of, the user knows what to do just by looking' (1990: 9). Poorly designed objects, so the message, would cause unnecessary problems for their users. With Norman, the concept of affordance was modified from Gibson's relational approach to accommodate design interests, suggesting that artifacts could be designed to suggest or determine certain forms of use through the notion of 'perceived affordances' (1990: 9). The question was no longer how organisms see, as was the case in Gibson's work, but rather how certain objects could be designed to encourage or constrain specific actions.

As others have noted (Maier et al., 2009; McGrenere and Ho, 2000; You and Chen, 2007), Norman's conceptualization of affordance became widely adopted within the design community, setting the standard for what would be considered good design and usability. For many designers and creative professionals, it was thus Norman's prescriptive formulation, not Gibson's work, which introduced them to the concept of affordance (You and Chen, 2007: 26). Unlike Gibson's relational understanding of affordance, Norman describes affordances as properties of things. Thus, power is placed in the hands of designers who have the power to enable and constrain certain action possibilities through their design choices. Whereas affordances may or may not be perceived in Gibson's view, according to Norman, the key is whether users actually perceive the intended possibilities for action inscribed into the design of an artifact. As Norman's concept of perceived affordances highlights, 'affordances are of little use if they are not visible to the users' (1999b: 41). This notion of perceived affordances has been hugely influential in professional user experience design and interface design, within the digital media and tech industries where Norman's framework still serves as the working definition of affordance. In their book Universal Principles of Design authors Lidwell et al. 
provide design guidelines and considerations through a number of concepts, including affordance, and closely follow Norman in suggesting that 'good' affordances can enhance the usability of a design and influence the way it is perceived (2010: 8-22).

\section{Technology affordances}

Affordances are not just limited to the visible senses but can also be felt or heard. As William Gaver suggests, 'affordances are primarily facts about action and interaction, not perception' (1996: 114). For example, 'when door handles are turned the sound of the latch may reveal the affordance of moving the door' (Gaver, 1991: 82-83). For Gaver, affordances are not just waiting to be perceived; rather they are there to be actively explored. Building on Gibson's relational model of affordances, Gaver contributed one of the first thorough explorations of affordances within the field of HCI with his paper on 'Technology Affordances' (Gaver, 1991; McGrenere and Ho, 2000). According to Gaver, affordances 'are properties of the world defined with respect to people's interaction with it' (1991: 80). It implies the 'complimentary of the acting organism and the acted-upon environment' (1991: 80). As Gaver exemplifies: 'whether a handle with particular dimensions will afford grasping depends on the grasper's height, hand size, etc. Similarly, a cat-door affords passage to a cat but not to me' (1991: 80).

Like Norman, Gaver thinks that technology and an artifact suggest different actions, but differs in his view that users know 'what to do just by looking' (Norman, 1988: 9). Gaver makes a separation between affordances and the perceptual information available about them, suggesting that affordances can be both perceptible and hidden (1991: 80). In the latter case, affordances must be inferred from other evidence, possibly through experimentation and other actions that make affordances visible. In the case of a graphical computer interface for example, a hidden affordance could be revealed by the action of a mouse-over. 
For Gaver, 'affordances exist not just for individual action, but for social interaction as well' (1996: 114). While the ecological approach to affordance stresses the relationship between the natural environment and the individual, 'how individual activities are shaped by the environment, and how individuals can orient to the relevant environmental attributes', Gaver is also concerned with 'the possibilities offered by the physical environment for social interaction' (1996: 114). He points out, 'these are not social affordances [...] but affordances for sociality' (1996: 114). In writing about the material features of technology, Gaver speculates about the differences between paper and electronic media, arguing that different affordances may have many effects on the social conventions that surround them. Contemplating the role of email, Gaver describes how moving from a high-bandwidth environment to a dial-up service dramatically changed the use and culture of email. 'The properties of email systems may not determine the communities that eventually form around them', Gaver suggests, 'but they do strongly constrain the cultures that might develop' (1996: 120). Although Gaver makes sure to distance himself from accusations of technological determinism, the term technology affordances establishes material qualities of technology as (partly) constitutive of sociality and communicative actions in this view. Again, we need to recognize the particular context and aim of Gaver's conception of affordance. Whereas Gibson's aim was to propose a theory of visual perception and Norman's primary concern was to enhance good design, Gaver sought to 'challenge researchers to avoid the temptation of ascribing social behavior to arbitrary customs and practices, and to focus instead on discovering the possibly complex environmental factors shaping social interaction' (1996: 125). In doing so, Gaver considered the concept of affordances as a 'useful tool for user-centered analyses of technologies' (1991: 97).

\section{Social affordances}

Gaver's aim of pointing researchers to the ways in which 'social activities are embedded in and shaped by the material environment' (1996: 125) might have paid of as many sociologists and 
communication scholars have subsequently used the notion of social affordances to refer to 'the possibilities that technological changes afford for social relations and social structure' (Wellman, 2001: 228) to talk about the ways in which 'technology affords social practice' (Hsieh, 2012). The term social affordance, however, might also seem confusing as it is primarily used to talk about the impact of technology, how technological properties enable and constrain sociality in certain ways. Social affordances are 'the social structures that take shape in association with a given technical structure' (Postigo, 2016: 5). Wellman et al. (2003) use the term to talk about the ways in which the internet may influence everyday life. Similar to how Gaver uses the notion of technology affordances to describe the changes in email culture effectuated by moving from high-bandwidth environment to a slower dial-up service, Wellman et al. (2003) use the notion of social affordance to describe how changes in broadband create new possibilities for communication. Using opposite prefixes, they both seem to be referring to the way in which technology affords sociality.

However, the notion of social affordance can also be understood in a much more general or relational sense without necessarily invoking technology explicitly. According to Gibson, 'the richest and most elaborate affordances of the environment are provided by other animals and, for us, other people' (2015: 126). This is to say that how people behave, move, or simply exist in an environment afford important cues as to how others should behave, move or co-exist. Take the pedestrian sidewalk; people constantly adjust their own movements to other people in their pathway. While the environment may give structure to an organisms' existence, Gibson suggests that 'behavior affords behavior' (2015: 127). Importantly, 'what the other animal affords the observer is not only behavior but also social interaction' (2015: 36). 


\section{Communicative affordance}

Characteristic of many of the above conceptualizations of affordances is the question of how technology and society relate. For the sociologist Ian Hutchby (2001a, 2001b) the term affordance provides a way to move beyond naïve technological determinism and strict social constructivism. Hutchby suggests that affordance provides a middle term that both takes into account the ways in which technologies are socially constructed and situated on the one hand, and materially constraining and enabling on the other hand. Specifically, Hutchby develops the concept of 'communicative affordances' referring to the 'possibilities for action that emerge from [...] given technological forms' (2001a: 30 ). This definition emphasizes how affordances are both functional and relational; 'functional in the sense that they are enabling, as well as constraining' and relational in terms of drawing 'attention to the way that the affordances of an object may be different for one species than for another' (Hutchby and Barnett, 2005: 151 emphasis in the original). Moreover, 'affordances can also shape the conditions of possibility associated with an action: it may be possible to do it one way, but not another' (Hutchby and Barnett, 2005: 151).

For Hutchby, the notion of communicative affordances is best understood as part of ordinary actions. While Gibson once observed that 'the walk-on-ability of a surface exists whether or not the animal walks on it', Hutchby and Barnett think 'it is nevertheless the case that the surface's affordance of walk-on-ability becomes manifest when the animal walks on it' (2005: 152). In other words, affordances can best be observed in the course of agential actions. In the context of media and communications research, the actions that seem particularly apt to study are the ones that imply communication of some sort. Hence the relative usefulness of the term communicative affordances as opposed to the very similar term social affordances. While both of these terms describe the ways in which technology enable or constrain social action, the term 
communicative affordances focuses specifically on the 'impact of technology for communication' (Schrock, 2015: 1233).

The term communicative affordances has most notably been used in research on mobile media (Boase, 2008; Helles, 2013; Hutchby and Barnett, 2005) as a way of describing how mobile devices 'alter communicative practices or habits' (Schrock, 2015: 1232). While the mobile phone has often been described as a device for 'perpetual contact' (Katz and Aakhus, 2002), the affordance of availability on this account first and foremost alters the degrees and ways of being available. In terms of mobility or portability, Helles usefully suggests that 'the central affordance of mobile phones is not the mobility of the device per se, but rather the fact that the user becomes a mobile terminus for mediated communicative interaction across the various contexts of daily life' (2013: 14). As Humphreys (2005) showed in her work on mobile use in public, the range of social contexts in which mobile communication takes place afford new forms of social identity, as well as the modification of tacit codes of social interactions. The communicative affordance perspective thus moves away from Norman's applied perspective of seeing affordances as features and instead focuses on more high-level abstractions of what mobile devices afford.

\section{Social media research and affordances}

As a concept that captures the relationship between the materiality of media and human agency, affordance continues to play an important role in media studies and social media research specifically (see Ellison and Vitak, 2015). Work in this area often uses an affordance approach to focus attention not on any particular technology, but on the new dynamics or types of communicative practices and social interactions that various features afford. Whereas some scholars have used affordance almost synonymously with the features of technology (Graves, 2007), others have focused on the social structures that are formed in and through a given 
technology (Baym, 2010; boyd, 2011; Postigo, 2016). Within the literature, social network sites and social media have often been analysed in terms of having 'affordances and constraints' (e.g. Baym, 2010; boyd, 2011; Ellison and Vitak, 2015).

\section{High-level and low-level affordances}

Affordances tend to be conceptualized on either one of two distinct dimensions: an abstract high-level or a more concrete feature-oriented low-level. High-level affordances are the kinds of dynamics and conditions enabled by technical devices, platforms and media. As danah boyd argues in her work on social network sites as a form of networked publics (2011: 39), these sites are essentially shaped by four central affordances: persistence, replicability, scalability, and searchability (2011: 46). For boyd, these high-level affordances of SNSs structure the engagement of users in these environments (2011: 39-40). Comparably, Treem and Leonardi (2012) allocate four affordances of social media use shaping organizational communication processes: visibility, editability, persistence, and association. Looking at what kind of behaviours social media afford offers 'one way researchers can transcend the particularities of any technology or its features, and focus on communicative outcomes' (Treem and Leonardi, 2012: 147). Schrock also sees affordances as something much broader than 'buttons, screens and operating systems' (2015: 1233). For him, affordances exist on a higher-level, as exemplified through the kinds of dynamics or conditions enabled by the mobile phone. For Schrock these affordances include: portability, availability, locatability and mulimediality (2015: 1229). In other words, what mobile media afford has nothing to do with a specific button, but rather with the kinds of communicative practices and habits they enable or constrain. In contrast, low-level affordances are typically located in the materiality of the medium, in specific features, buttons, screens and platforms. Interestingly, we find that many researchers use this low-level or design-oriented notion of affordance more in passing than in an analytical sense. As such affordance becomes a way of talking about the technical features of a platform, 
for example, invoking the term to describe Twitter's 140-character limit or the ability to share a link using a tweet button. In a Gibsonian sense, technical features — understood as the furniture of the digital landscape - afford certain actions such as clicking, sharing, or liking.

More often than not, however, authors seem to combine a high-level understanding of affordance with a consideration of specific features or platforms. As boyd argues in her work, higher-level affordances are conditioned by the properties of bits, which essentially introduce new opportunities for interaction and communication (2011: 39). Comparably, Ellison and Vitak (2015) advocate for considering the high-level affordances of social media by conjoining this perspective with looking at specific features such as the profile to understand social capital processes. Conversely, Postigo uses a low-level or feature-oriented conceptualization of affordance as a means of studying how YouTube's platform features create 'a set of probable uses/meanings/practices for users while serving YouTube's business interests' (2016: 332). Postigo thus starts his analysis by describing a set of architectural features and properties of the YouTube platform in order to describe its 'social affordances' (2016: 336).

While all conceptualizations of affordance take Gibson's original framing of the term as a starting point, they differ in terms of where and when they see affordances materializing (i.e. features, artifacts, social structures) and what affordances are supposed to activate or limit (i.e. particular communicative practices, sociality, publics, perception). By highlighting the difference between high-level affordances and low-level affordances, we want to emphasize what we see as one of the main ontological and epistemological differences in how the concept of affordance gets used. The Gibsonian understanding locates affordances in the relation between a body and its environment, whereas the low-level conception of affordance takes on after Norman by locating affordances in the technical features of the user interface (Sun and Hart-Davidson, 2014: 3537). 


\section{Imagined affordances}

Despite the popularity and widespread usage of the term in social media research, recent contributions to the literature on affordance have called for more precise and nuanced definitions of the term, variously proposing additional concepts to better account for the complex relationships between technology and sociality (see Ilten, 2015; McVeigh-Schultz and Baym, 2015; Nagy and Neff, 2015). Nagy and Neff, for instance, have introduced the notion of 'imagined affordance' as 'a theory that better incorporates the material, the mediated, and the emotional aspects of human-technology interaction' (2015: 2). Imagined affordances, Nagy and Neff contend, 'emerge between users' perceptions, attitudes, and expectations; between the materiality and functionality of technologies; and between the intentions and perceptions of designers' (2015: 5). For Nagy and Neff 'older' notions of affordance fail to address the complexity of cognitive as well as emotional processes. Simply locating the action possibilities of a social media platform in a set of features will not do, they claim, because users' perceptions, beliefs, and expectations of what the technology does or what the platform suggests it is for 'shape how they approach them and what actions they think are suggested' (2015: 5). Imagined affordances may not just affect how users approach social media platforms, they may performatively help to shape the platforms themselves. As Bucher (2016) argues in her research on users' understanding and perceptions of algorithms in social media platforms, the ways in which users imagine and expect certain algorithmic affordances, affect how they approach these platforms. The feedback-loop characteristics of machine learning systems like Facebook make user beliefs an important component in shaping the overall system behaviour, as end-user activity is generative of the system itself. 


\section{Vernacular affordances}

In a similar vein, McVeigh-Schultz and Baym (2015) suggest linking the materiality of social media platforms to the sense-making processes of users when conceptualizing affordances. Developing the notion of 'vernacular affordance', McVeigh-Schultz and Baym emphasize 'how people themselves understand affordances in their encounters with technology’ (2015: 1). Instead of researchers or designers assigning affordances to social media, they derive the affordances from users discussing how they engage with the technology. Such a perspective, they state, infers affordances from a more 'vernacular' account of the users themselves who may emphasize different aspects of the action possibilities of a platform. Interviewing key actors of NGOs involved in a campaign entitled 'Free Lunch for Children in China' (FL4C) on the Chinese microblogging platform Sina Weibo, researchers Zheng and $\mathrm{Yu}$ found that Weibo affords NGOs to scale their network, frame collective action, and establish the legitimacy of their campaign (2016). Schools participating in the FL4C campaign had to post photographic evidence of the lunches provided to Weibo, thereby 'enforcing transparency,' 'enhancing accountability,' and 'enacting public scrutiny' (Zheng and Yu, 2016: 305), affordances which only revealed themselves through interviews and observing the practices of the actors involved in the campaign. In line with audience research more generally, the point with emphasizing the notion of vernacular affordances is to show how action possibilities cannot be determined once and for all, but need to be grounded in people's own perceptions and experiences. As McVeighSchultz and Baym argue in their study of how people use and make sense of the dating app Couple, 'affordances are not experienced in isolation, but rather in relation to a complex ecology of other tools with other affordances' (2015: 2). Thus, affordances simultaneously exist for people at multiple levels and across platform boundaries. 


\section{Re-assembling affordances}

Our purpose with outlining the different conceptions of affordance was to point out its different intellectual history, ontological status and analytical value. It arguably makes a difference which conception of affordance is used-as it puts certain epistemological limits on what can be known about affordances and where to find them in the first place. When designers and designed artifacts are given priority, affordances are seen as part of the technological design. Following an understanding of affordance as communicative, however, locates affordances as part of communicative actions and can best studied in and through the kinds of practices that technology allows for or constrains. Or, in the case of vernacular affordances, ways of knowing and analysing affordances requires an emphasis on users' own accounts of technology, where affordances become as much part of users' experiences and perceptions of technologies as of the technologies themselves.

\section{A relational and multi-layered approach to affordances}

The different concepts outlined above seem to focus on what technology does to users, and not for instance the other way around. Given the relational ontology of Gibson's original concept, it seems somewhat surprising that the relationality in question often seems to be applied rather unidirectional. The question is seldom what end-users afford or do to technology (not to be confused with the question of what users do with technology), or what platforms afford to other kinds of users beside end-users. In order to do the concept of affordance justice we need to think much more relationally and multi-layered about the concept, while retaining a sense of platform-sensitivity by taking the medium-specificity of platforms into account. ${ }^{10}$ If ActorNetwork Theory (ANT) and similar relational approaches to the relationship between technology and the social have taught us anything, isn't it to think more fully-fledged about agency and connectivity? ANT-while not a coherent approach-holds that agency is distributed and relational, and that nonhumans are actors with agency too. As Latour suggests, the agency 
of nonhumans refers to the ways in which 'things might authorize, allow, afford, encourage, permit, suggest, influence, block, render possible, forbid, and so on' (2005: 72 emphasis added). As Latour acknowledged in a footnote to this much-cited reference on the idea of nonhuman agency, it is highly indebted to Gibson's notion of affordances and the question of what technology does to users. This may also be where some of the overemphasis on affordances as something seemingly tied to the agency of technological objects comes from. While the notion of nonhuman agency is important in studying social media platforms, we should also not lose sight of the multi-directionality of agency and connectivity at work in approaching questions of affordances. If one way of thinking more relationally about affordances on par with Gibson's original conception means considering agency as pointing 'both ways, to the environment and to the observer' (2015: 121), then we want to suggest that thinking more multi-layered about affordances means reconsidering the notion of the interface as well. Although providing a genealogy of the notion of the interface falls outside the scope of this chapter, let it suffice at this point to briefly explain what we mean by interface and why it is important to our current discussions.

While Gibson saw affordance as manifest in the surfaces and layout of the terrestrial environment, design studies and HCI in many ways have treated this as being analogous to graphical user interfaces, button and features in the context of digital environments. Following Branden Hookway (2014), however, we want to argue that surfaces and interfaces are not necessarily the same. A surface, Hookway suggests, 'exists primarily as an aspect of that which it surfaces'; it means 'a facing above' (2014: 12). The interface, by contrast, 'does not primarily refer back to a thing or condition but rather to a relation between things or conditions, or to a condition as it is produced by a relation' (Hookway, 2014: 13-14). While we do not want to go as far as Hookway in replacing all references to a web interface and the vernacular understanding of interface as the place of interaction between two systems with the term surface, we also want to stress that interfaces are not confined to this notion of a surface or a 
physical boundary of sorts. Following Hookway, interfaces should above all be understood as 'forms of relations'. If interfaces are not simply surfaces or computer screens, what does it mean to consider interfaces as sites of analysis for a study of affordances? Previously, Mel Stanfill has proposed a method for analysing websites entitled 'discursive interface analysis' which takes a site's affordances as a starting point in order to analyse how they produce and make visible particular norms of use (2014: 3). However, following Hookway (2014) we wish to emphasize how interfaces produce and make visible particular relations. By moving beyond the surface and to platforms' distinct interfaces that mediate between different parties, we wish to draw attention to how affordances do not only present and structure action possibilities for end-users in the graphical user interface, but also how 'the interface privileges the question of how a relation may come into being and how it may produce behaviors or actions' (Hookway, 2014: 14). By approaching the question of affordance from a relational and multi-layered perspective, the question is not just whose action possibilities we are talking about, but also how those action possibilities come into existence by drawing together (sometimes incompatible) entities into new forms of meaningfulness. That is, there is nothing that necessarily warrants the analogous treatment of the Gibsonian animal or observer with the end-user. Correspondingly, we might wonder where in the course of the historical trajectory the properties of the natural environment that Gibson describes became synonymous with devices, technologies and the graphical userinterface?

\section{Platforms as environments}

If the end-user is not necessarily equivalent to the Gibsonian animal, then who or what constitutes the animals of social media and how are we to think of the environment in this case? For Gibson, the terrestrial environment with its terrestrial features of paths, cliffs, barriers, water margins etc. afford various ways of existing relative to the animal in question. In Gibson's terms, the 'earth has "furniture”...it is cluttered' (2015: 123): 'Like the furnishings of a room', 
the environment is cluttered with objects and features, 'it is what makes it liveable' (2015: 71). For us, social media platforms constitute a form of environment too, composed of pathways and features in their own right. It is important to point out however, that no two platforms are alike though many tend to use similar features and functionalities such as likes, shares, comments or hashtags. While we focus specifically on Twitter as a platform in this chapter, and take its platform-specificity into account, the broader arguments about considering platforms as environments with its specific possibilities and constraints holds true for any social media platform of study.

Platforms, in their computational understanding, are seen as infrastructures that can be programmed and built on (Bogost and Montfort, 2009). In the context of Twitter, this programmability is facilitated by application programming interfaces (APIs), which enable interoperability and facilitate third-parties to employ platform data and functionality (Bodle, 2011; Bucher, 2013; Helmond, 2015). At the same time APIs enable platforms to extend into the web by setting up data channels that make external web data flowing back to their databases 'platform ready' in order to fit the underlying economic business models of social media platforms (Helmond, 2015). Platforms are technologies or services that mediate interactions and relations between two or more parties (Rochet and Tirole, 2003), and social media platforms can be characterized as digital intermediaries that draw together and negotiate between different stakeholders such as end-users, developers and advertisers which each come with their own aims and agendas (Gillespie, 2010). Social media platforms are characterized by the combination of their infrastructural model as programmable and extendable infrastructures, and their economic model of connecting end-users to advertisers (Helmond, 2015) where the 'politics of platforms' lies in the mediation of these stakeholder relations (Gillespie, 2010). For example, Facebook's Like button enables Facebook to extend into websites and apps, where the data produced on and collected through these external sources is sent back to the platform 
(Gerlitz and Helmond, 2013) and made available to advertisers through various advertising interfaces such as the Facebook Marketing API. ${ }^{11}$

\section{A platform-sensitive approach}

If platforms are specific kinds of digital environments, and affordances reflect the complex coevolution of users and environment, how are we to make sense of such contextual relationality in the concrete case of a social media platform? In the following we take the example of Twitter as a case in point to discuss what we term a platform-sensitive approach to the affordances of social media.

\section{The case of Twitter platform changes}

Exactly ten years ago today, on March 21 ${ }^{\text {st }}, 2006$, Twitter's cofounder Jack Dorsey published the first tweet ever. ${ }^{12}$ Since then, Twitter has grown into the most popular microblogging platform to date with 320 million monthly users (Styles, 2016). Still, the alleged 'decay' of Twitter has been regularly pronounced, particularly in light of its loss of 'sociability' (Burgess, 2015), stalled user growth, and the many recent platform changes (see Topolsky, 2016; Tsukayama, 2016). Commentators have noted how declining user growth has prompted the platform to change some of its most prevalent features and functionalities, including the favorite feature as briefly discussed in the introduction of this chapter. When, in November 2015, the favorite was changed into a like it was indeed presented as a way of making the service more comprehensible to new users (Kumar, 2015). The turn to hearts, or likes, was also motivated by a desire to standardize expressions across all Twitter products as exemplified by Twitter's spokesperson who put it in this way: 'You've embraced hearts in a big way on Periscope, and we're delighted to bring them to Twitter and Vine, making them the common language for our global community' (Kumar, 2015). A few months later, in February 2016, Twitter made another 
major change by enabling a new timeline ordering in which end-users would now receive "the best Tweets first' by default in order to 'catch up on the best Tweets from people you follow' (About your Twitter timeline, n.d.). This change essentially transformed the curation and presentation of the feed, from that of a linear progression of tweets in a reverse-chronological order to a feed no longer exclusively organized around a linear flow of time. With the new feed introduced early 2016, tweets would now be organized around various popularity and affinity metrics, including user interaction and their engagement with tweets through platform activities such as liking, retweeting and replying (About your Twitter timeline, n.d.). These two controversial changes were heavily discussed on technology blogs as well as on Twitter itself, where the rumoured introduction of what came to be widely discussed as 'the algorithmic timeline' of Twitter, prompted much outrage under the hashtag \#RIPTwitter. ${ }^{13}$ The question is what, if anything, these incidents have to do with affordances? As we will argue, much, indeed.

At first sight it might be tempting to interpret Twitter's claim that the favorite button is confusing to use, by evoking Norman's idea of good interface design. From a design-centred perspective the favorite button failed to communicate how the feature should to be used. The turn to hearts and likes could be read as an attempt to enhance the perceived affordances of the feature by indicating more clearly 'how the user is to interact with the device' (Norman, 1990: 8). However, if we tackle the question from the perspective of communicative or vernacular affordances it quickly becomes apparent that affordances cannot simply be understood in terms of good or bad design. What the many reactions to the change and previous studies attest to is that the favorite feature has been attributed various affordances beyond their original purpose of saving tweets you like (Stone, 2006). In their study of Twitter favoriting behaviour, Meier et al. (2014) identified 25 distinct motivations for using favorites including showing agreement and relationship building. From the perspective of end-users, the favorite feature does not simply afford bookmarking a tweet, it also affords different types of sociality and communicative actions, e.g. favoriting as a form of communication to maintain relations, to show agreement, or 
to indicate the end of a conversation (cf. Meier et al., 2014). The change to hearts and likes, while technically not affording any new actions, certainly seems to suggest different communicative and social affordances. For some end-users, the change from a favorite into a like feature eradicated the wide range of 'communicative affordances' previously associated with the favorite (Newton, 2015). Others still, claimed the opposite, suggesting the importance of taking users' perspectives into account when analysing the affordances of social media. Simply approaching the favourite and like features on Twitter as examples of technical affordances will not do. While features clearly afford actions, only looking at what features allow users to do would quickly miss out on the multifarious meanings and communicative affordances these buttons entail. Although technically affording identical actions, taking into account what users say, believe and expect these features to be able to accomplish varies greatly.

Similar points could be made when considering the recent introduction of Twitter's 'algorithmic timeline' and the reactions that followed, particularly around the \#RIPTwitter trending topic. Here, assessing affordances in terms of whether the Twitter feed is being used in the ways envisioned by designers in the vein of Norman would fail to acknowledge "the role of the Twitter hashtag as a means of coordinating a distributed discussion among large numbers of users' and to explain the emergence of such 'hashtag publics' (Bruns and Burgess, 2015: 14). What a concept like imagined affordances usefully points to is that we also need to consider the ways in which the technology is imagined by its users (Nagy and Neff, 2015: 4). As Bucher (2016) has shown in her research on how Facebook's algorithms are imagined, people's expectations and perceptions of technology may affect behaviour as much as its material properties may do. Similarly, the \#RIPTwitter reveals how multiple imaginings exist around what an algorithm is for, and what the prospects of an algorithmically curated timeline would do to the possibilities and constraints of Twitter. 
Rather than introduce yet another concept of affordance, our aim in the remaining part is to contribute one way of approaching the empirical analysis of affordances in social media by being sensitive to platform specificities. This specificity entails not merely looking at features that manifest on a graphical user interface, but considers how the affordances of platforms are relational and multi-layered.

\section{Expanding the notion of the user}

Taking the specificity of platforms into account requires extending our notion of the 'user' as platforms cater to different users, actors, or stakeholders. We argue for moving beyond the enduser-centred (e.g. Gaver, 1991: 97; Gibson, 2015) and designer-centred (e.g. Norman, 1990) approaches that have figured prominently within affordance theory. In the literature, the term 'user' is often used synonymously with the human end-user, the person for whom the website or app was originally designed. However, advertisers, developers, and researchers are platform users in their own right too. Arguably, Twitter affords different things to an end-user than to a developer, advertiser or researcher. These different user types are predefined by Twitter, by addressing them via distinct interfaces such as the end-user interfaces including the website and Twitter apps, the Twitter APIs for developers and researchers, and the Advertising API for advertisers. In predefining these users, they could be considered the intended or designed-for user types. While end-users typically stress the communicative or social affordances associated with certain features available on the graphical user interface of Twitter, most developers would probably stress the importance of another interface altogether.

For developers, the action possibilities of Twitter are primarily related to its APIs, which affords them to build applications on top of the platform (Bucher, 2013). APIs afford developers, but also researchers, ${ }^{14}$ advertisers and other users, access to and the possibility to re-use the data and functionality of platforms. Using the Twitter APIs, ${ }^{15}$ a number of third-party services have been 
built on top of Twitter, including services like Favrd and Favstar, which have utilized Twitter's favorite feature. These services have made the act of favoriting public by aggregating favorite tweets, and by showing users who had favorited their tweets (MacManus, 2009). In doing so, Favrd turned the favorite from a personal bookmarking mechanism into a public popularity measure (MacManus, 2009). Favstar further enhanced the public nature of favorites by offering a social network around favorites through leader boards and other features (Paßmann et al., 2014). In this way, developers - in conjunction with end-users and their use practices — have played an important role in shaping the action possibilities of the favorite button by envisioning new use case scenarios. The creation of third-party services built on top of the favorite feature, such as Favstar, shows how developers may conceive of new action possibilities which in their turn enable novel communicational dynamics outside of the Twitter platform. It emphasizes the specificity of Twitter, as a platform which has a history of being fairly 'open' for third-party development, as an environment in which the generative potentials of end-users and developers should be taken into account when examining the platform's affordances.

In light of stalled user growth at Twitter, the change from favorites to likes should not just be seen as an attempt at catering to end-users' needs and practices (i.e. 'you've embraced hearts' as Twitter's spokesperson has said), but also as an integrated approach to the standardisation of expressions that enables or operates according to a dual logic of decentralisation and recentralisation that extends the 'environment' beyond the confines of the platform (Helmond, 2015). The Twitter APIs and their underlying logic of connectivity have enabled an ecosystem of third-party apps and services, weaving Twitter into the larger social media ecosystem (Van Dijck 2013). ${ }^{16}$ This includes creating connections between Twitter and other Twitter-owned services such as Periscope and Vine, as well as other social media platforms, e.g. through the practice of automatically cross-posting content to Facebook or LinkedIn, thereby extending Twitter's functionalities into the larger social media ecosystem. Furthermore, the change from favorite into like can be seen as a form of standardizing modes of engagement across various 
social media platforms, or as technology reporter Newton notes: 'likes and hearts have become a kind of universal currency of the social web, from Facebook to Tumblr to Instagram' (2015). The like, as a 'universal currency' in social media, is particularly important to advertisers, for whom the Twitter platform affords the promotion of content by targeting end-users and the measurement of engagement. While the like feature has certain communicative affordances such as signalling support or sharing information with friends (Brandtzaeg and Haugstveit, 2014; Guy et al., 2016), for advertisers the feature and the actions it enables facilitates possibilities to tailor and personalize ads, and to measure engagement. Twitter's Ads API ${ }^{17}$ connects Twitter, Twitter Official Partners ${ }^{18}$ and advertisers to target end-users based on who they follow, information about the device they are using such as IP address and installed apps, and interactions with tweets including retweeting, replying and liking amongst other signals. ${ }^{19}$ These activities are also used to predict the engagement rate for an ad in order to determine its relevancy for a user, and subsequently the ad's price. ${ }^{20}$ Moreover, Twitter collects data from people engaging within the wider 'Twitter ecosystem' (Twitter Help Center, n.d.), by tracking end-users and non-Twitter users across the web via platform functionality such as Twitter buttons on external websites and apps (cf. Gerlitz and Helmond, 2013). This is what Heyman (2016: 143) calls the 'hidden affordances' (as defined by Gaver) of social media platforms since users do not perceive the action possibility of placing cookies to track users across the web.

\section{Platform users and their interfaces}

If the users of platforms are diverse and multiple, so are their points of contact. Users can form relations with the platform through multiple interfaces (or surfaces in Hookway's notion) such as the various APIs that have been discussed above. However, these are not isolated or separate points of contact between the Twitter platform and its different users. Rather, together they constitute the platform environment as a whole and enable relations between all users inside the platform, as well as relations with users outside of the platform's boundaries. Similar to the 
ways in which Gibson foregrounds the relationality between an animal and the environment in his conceptualization of affordance, Hookway's notion of interface privileges the formation of a relation, and how that relation in turn may become productive of certain actions. Social media platforms and their underlying logic of 'connectivity' (Van Dijck, 2013) also require a view on affordances that moves beyond the boundaries of the platform and takes the larger environment the platform operates in into account. The notion of connectivity, José Van Dijck and Thomas Poell contend, refers to 'the socio-technical affordance of networked platforms to connect content to user activities and advertisers' (2013: 8). In the case of the Twitter platform and its users, the different APIs may provide some clues as to how different relationships come into being and the kinds of actions that are enabled. Importantly, these APIs also transform the domain and practices of advertising, research and marketing themselves insofar as they are now being channelled through Twitter. As our brief discussion of interfaces has shown, platforms address users differently and the possibilities for action are therefore relative to users (see Gibson, 2015; Hutchby, 2001). The move from favorite to likes, we want to suggest, is symptomatic of this drive towards connecting various users together by standardizing the metric of endorsement across all Twitter products and places connected to those products (such as external websites or apps).

\section{The adaptability of platform surfaces}

In addition to expanding the notion of the user and looking at different interfaces, a third important aspect of a platform-sensitive approach is to consider the specificity of the digital environment more explicitly. What distinguishes social media platforms from Gibson's natural environment is the increasingly dynamic and malleable nature of these platforms. Following Gibson, platforms present themselves relative to their inhabitants, but contra Gibson, for whom the surfaces of the natural environment do not change as the needs of the observer changes, social media platforms adapt their surfaces to their users. These surfaces are optimized for web 
or mobile use and they can be adapted through platform settings and personalization features, which create personalized surfaces for end-users. In addition, the common practice of $\mathrm{A} / \mathrm{B}$ testing, a technique to test the effectiveness of new features, functionalities or algorithmic recommendations with different groups of end-users of the platform, generates multiple platform surfaces. Platform surfaces are relative because they, in many cases, are personalized for each end-user and because platforms create and present multiple interfaces (as discussed in the previous section) that enable and constrain users' action possibilities.

A key question that we think social media researchers should be asking in terms of affordance theory is how the environment they are studying differs from the kind of environment studied by Gibson and other affordance theorists. When Gibson wrote about what the environment affords the animal, he was primarily thinking of its terrestrial features such as water margins, rocks and cliffs (2015: 31-36). A cliff offers certain things because of what it is, not because of the wishes bestowed upon it by an interlocutor (Gibson, 2015: 130). With the computational systems driving social media platforms, the environment takes on a different character that we think needs to be attended to. More specifically, it seems that an algorithmically organized environment challenges the invariant nature of affordances in important ways. As noted by Nagy and Neff, technologies are increasingly 'adaptive, learning, responsive, and changing along with the users they share an environment with' (2015: 5). While Facebook has often been the exemplary case in terms of the adaptable and malleable nature of platforms, the move towards non-linear news feeds on platforms such as Twitter and Instagram ${ }^{21}$ suggests a more general tendency towards a personalized furnishing (to use Gibson's words) of the social media environment. While the term 'algorithmic timeline' may be somewhat of a misnomer for a feed that technically speaking is already algorithmic in the sense that tweets are arranged in a reverse-chronological manner by an algorithm, the extent to which algorithms are used to organize feeds relative to users and their activities is new. This means that the digital environment does not merely offer something to its users, users' needs and individual likings 
and behaviours increasingly play a generative role in producing those very offerings in the first place.

(Non)human agency affording things to technology

The feedback-loops and generative role of users in shaping the algorithmically entangled social media environment brings us to our final point we want to make in terms of what we mean by a platform-sensitive approach to affordances. While affordance theory has mainly put emphasis on the question of what technology affords users, the socio-technical nature of social media platforms also begs the reverse question of what users afford to platforms? In the age of socalled big data fuelled by expansive data points and machine learning algorithms which 'can learn from and make predictions about data without being explicitly programmed to do so, ${ }^{, 2}$ and respond to user input in an adaptive way. For example, Facebook uses machine learning for ranking search results, serving the most relevant ads, identifying faces, and predicting which memories people would like to view (Applied Machine Learning, n.d.). The platform feature 'On This Day' employs a machine learning model which learns in real time and continuously from people about which memories they would like to see in their News Feeds (Aziz and Paluri, 2016). In these cases, the question of who affords what, to whom seems increasingly more complex.

In order to do the relational view on affordances full justice, we need to consider the multidirectionality of agency and connectivity at play. More specifically this means looking at what users afford to the environment, where users are understood in the expanded and typified ways explicated above. We have not been able to account for all the different forms of users above (nor do we believe such an exhaustive account is even possible), but want to stress that users are not confined to humans such as end-users and developers, but to nonhumans alike. As actors in 
their own right, algorithms can be considered 'hidden affordances' in Gaver's sense as their action possibilities shape our social relations and the content we see without being directly perceivable as such (Nagy and Neff, 2015: 3). Moreover, it's not only a question of what these algorithms afford to end-users but also what they afford to the platform. In sorting and ranking content by displaying 'the best Tweets first' based on 'accounts you interact with most, Tweets you engage with, and much more' in order to 'catch up on the best Tweets from people you follow' (About your Twitter timeline, n.d.), algorithms are geared towards the creation and maintenance of participation and engagement. In light of Twitter's stalled user growth, the move towards an algorithmically sorted timeline can be interpreted as a corporate attempt at becoming more relevant, and therefore more engaging for its users.

As we see it then, a platform-sensitive approach requires a socio-technical sensibility towards the distributed agency of humans and nonhumans at play. As Gibson himself pointed out, affordance 'cuts across the dichotomy of subjective-objective' (2015: 121). Rather than thinking of the affordances of social media platform as one-way relationships whereby either the technology affords something to users, or as we might have suggested in this section, users afford things to technology, the presence of algorithms in particular suggests that such unidirectionality does not hold. By clicking and liking end-users fuel the algorithms, which in their turn generate the information flows fed back to end-users. Moreover, the clicks and likes fuel the interest and engagement of developers, researchers and advertisers who help to keep the platforms in business.

Furthermore, the specific affordances of Twitter as an extendable platform, which includes Twitter's extension into the web and the app space through the Twitter button and Sign in with Twitter login feature, feeds data such as likes generated in external websites and third-party apps back into the platform. When Twitter end-users are browsing websites outside of Twitter 
with Twitter Login or a Twitter button, they are still part of the 'Twitter ecosystem., ${ }^{23}$ Data about these website and app visits in the Twitter ecosystem flows back into the Twitter platform and is used to profile users based on the websites they have visited to suggest new people to follow who visit similar webpages. In other words, we might see how the activities of a diverse set of users both inside as well as outside of the platform's boundaries, afford back to the platform. Thus, a platform-sensitive approach to affordances should consider how a platform's infrastructure extends its affordances beyond its own environment and how they may be integrated in other platforms and services as well as how these activities afford back to the platform and its multiple users.

\section{Conclusion}

The notion of a platform-sensitive framework is meant to emphasize the specificity of platforms as a socio-technological environment that draw different users together and which orchestrate the relations between different platform users. Such a perspective enables us to see how platforms may afford different things to various types of users, including end-users, developers, and advertisers; and considers how they are connected through various possibilities for action. As demonstrated in our case study on two recent platform changes, the like provides distinct action possibilities for end-users (e.g. liking a tweet) and to advertisers (e.g. measuring engagement), enabling us to comprehend affordances vis-à-vis the economic underpinnings of platforms. In addition, we have emphasized how affordances extend across the boundaries of platforms and how both human and nonhuman users importantly afford something back to the platform. In the case of Twitter's new algorithmic timeline, it does not only afford end-users to receive relevant content first but this relevance is in its turn established by those same end-users liking, replying and retweeting content thereby affording things back to the algorithm and the platform. Thus, employing the concept of affordance to study social media requires explicating how the concept is used and considering the work it may do analytically. Furthermore, for the 
study of social media platforms, as we have argued throughout this chapter, we should take the specificity of platforms into account which requires us to revisit earlier conceptualizations of affordance and to critically analyze how they may be employed or translated to platform environments. 


\section{References}

About your Twitter timeline (n.d.) Twitter Help Center. Available from:

https://support.twitter.com/articles/164083 (accessed 10 February 2016).

Applied Machine Learning (n.d.) Research at Facebook. Available from:

https://research.facebook.com/machinelearning (accessed 5 June 2016).

Aziz O and Paluri M (2016) Engineering for nostalgia: Building a personalized 'On This Day' experience. Facebook Code. Available from:

https://code.facebook.com/posts/1748968875380127/engineering-for-nostalgiabuilding-a-personalized-on-this-day-experience/ (accessed 5 June 2016).

Baym NK (2010) Personal Connections in the Digital Age. 1 edition. Cambridge, UK: Polity.

Boase J (2008) Personal Networks and the Personal Communication System. Information, Communication \& Society 11(4): 490-508.

Bodle R (2011) Regimes of Sharing. Information, Communication \& Society 14(3): 320-337.

Bogost I and Montfort N (2009) Platform Studies: Frequently Questioned Answers. In:

Proceedings of the Digital Arts and Culture Conference, University of California, Irvine. Available from: http://escholarship.org/uc/item/01r0k9br.pdf (accessed 2 September 2012).

boyd danah (2011) Social network sites as networked publics: Affordances, dynamics, and implications. In: Papacharissi Z (ed.), A Networked self: identity, community, and culture on social network sites, New York: Routledge, pp. 39-58.

Brandtzaeg PB and Haugstveit IM (2014) Facebook Likes: A Study of Liking Practices for Humanitarian Causes. International Journal of Web Based Communities 10(3): 258279. 
Bruns A and Burgess J (2015) Twitter Hashtags from Ad Hoc to Calculated Publics. In:

Rambukkana N (ed.), Hashtag Publics: The Power and Politics of Discursive Networks, New York: Peter Lang Pub Inc, pp. 13-28.

Bucher T (2013) Objects of Intense Feeling: The Case of the Twitter API. Computational Culture 3. Available from: http://computationalculture.net/article/objects-of-intensefeeling-the-case-of-the-twitter-api (accessed 6 May 2014).

Bucher T (2016) The algorithmic imaginary: exploring the ordinary affects of Facebook algorithms. Information, Communication \& Society 0(0): 1-15.

Burgess J (2015) Twitter (probably) isn't dying, but is it becoming less sociable? Medium. Available from: https://medium.com/dmrc-at-large/twitter-probably-isn-t-dying-but-isit-becoming-less-sociable-d768a9968982\#.jr4okd76o (accessed 20 May 2016).

Ellison NB and Vitak J (2015) Social Network Site Affordances and Their Relationship to Social Capital Processes. In: Sundar SS (ed.), The Handbook of the Psychology of Communication Technology, Boston: Wiley-Blackwell, pp. 205-227.

Gaver WW (1991) Technology affordances. In: Proceedings of the SIGCHI conference on Human factors in computing systems, ACM, pp. 79-84. Available from: http://dl.acm.org/citation.cfm?id=108856 (accessed 20 January 2016).

Gaver WW (1996) Situating action II: Affordances for interaction: The social is material for design. Ecological Psychology 8(2): 111-129.

Gerlitz C and Helmond A (2013) The Like economy: Social buttons and the data-intensive web. New Media \& Society 15(8): 1348-1365.

Gibson JJ (1982) Notes on affordances. In: Reasons for realism. Selected Essays of James J. Gibson. London: Lawrence Erlbaum Associates, pp. 401-418. 
Gibson JJ (2015) The Ecological Approach to Visual Perception. Classic Editions. New York: Psychology Press.

Gillespie T (2010) The politics of 'platforms'. New Media \& Society 12(3): 347-364.

Graves L (2007) The Affordances of Blogging: A Case Study in Culture and Technological Effects. Journal of Communication Inquiry 31(4): 331-346.

Guy I, Ronen I, Zwerdling N, et al. (2016) What is Your Organization 'Like'? A Study of Liking Activity in the Enterprise. In: CHI'16, San Jose, CA: ACM. Available from: https://www.research.ibm.com/haifa/dept/imt/papers/Liking.pdf (accessed 24 March 2016).

Helles R (2013) Mobile communication and intermediality. Mobile Media \& Communication 1(1): 14-19.

Helmond A (2015) The Platformization of the Web: Making Web Data Platform Ready. Social Media + Society 1(2). Available from: http://sms.sagepub.com/content/1/2/2056305115603080 (accessed 30 September 2015).

Heyman R (2016) Facebook \& Users: Who is Using Who? A Material Semiotic Approach to the Irreversibilisation of Facebook as a Case of Lifeworld Colonisation by Social Media. Ph.D., Brussels, Belgium: Vrije Universiteit Brussel.

Hookway B (2014) Interface. Cambridge, MA: MIT Press.

Hsieh YP (2012) Online social networking skills: The social affordances approach to digital inequality. First Monday 17(4). Available from:

http://firstmonday.org/article/view/3893/3192 (accessed 20 January 2016). 
Humphreys L (2005) Cellphones in public: social interactions in a wireless era. New Media \& Society 7(6): 810-833.

Hutchby I (2001a) Conversation and Technology: From the Telephone to the Internet. Cambridge, UK: Polity.

Hutchby I (2001b) Technologies, texts and affordances. Sociology 35(2): 441-456.

Hutchby I and Barnett S (2005) Aspects of the sequential organization of mobile phone conversation. Discourse Studies 7(2): 147-171.

Ilten C (2015) 'Use Your Skills to Solve This Challenge!': The Platform Affordances and Politics of Digital Microvolunteering. Social Media + Society 1(2). Available from: http://sms.sagepub.com/content/1/2/2056305115604175 (accessed 11 February 2016).

Katz JE and Aakhus M (2002) Perpetual contact: Mobile communication, private talk, public performance. Cambridge, UK: Cambridge University Press.

Kumar A (2015) Hearts on Twitter. Twitter Blogs. Available from: https://blog.twitter.com/2015/hearts-on-twitter (accessed 27 November 2015).

Langlois G (2014) Meaning in the Age of Social Media. New York: Palgrave Macmillan US. Available from: http://link.springer.com/10.1057/9781137356611 (accessed 5 June 2016).

Latour B (2005) Reassembling the Social: An Introduction to Actor-Network-Theory. Oxford, UK: Oxford University Press.

Lidwell W, Holden K and Butler J (2010) Universal principles of design, revised and updated: 125 ways to enhance usability, influence perception, increase appeal, make better design decisions, and teach through design. Second. Beverly: Rockport Publishers. 
Lunden I and Olanoff D (2015) Twitter CEO Dorsey Apologizes To Developers, Says He Wants To 'Reset' Relations. TechCrunch. Available from: http://social.techcrunch.com/2015/10/21/twitter-ceo-dorsey-apologizes-to-developerssays-he-wants-to-reset-relations/ (accessed 5 April 2016).

MacManus R (2009) Favrd Shuts Down - Not Twitter's Last Laugh (Thank you, Textism). ReadWrite. Available from: http://readwrite.com/2009/12/06/favrd_shuts_down_show_goes_on_thank_you_textism (accessed 25 January 2016).

Maier JR, Fadel GM and Battisto DG (2009) An affordance-based approach to architectural theory, design, and practice. Design Studies 30(4): 393-414.

McGrenere J and Ho W (2000) Affordances: Clarifying and evolving a concept. In: Proceedings of Graphics Interface 2000, Montréal, Québec, pp. 179-186. Available from: http://graphicsinterface.org/proceedings/gi2000/attachment/gi2000-24/ (accessed 20 January 2016).

McVeigh-Schultz J and Baym NK (2015) Thinking of You: Vernacular Affordance in the Context of the Microsocial Relationship App, Couple. Social Media + Society 1(2). Available from: http://sms.sagepub.com/content/1/2/2056305115604649 (accessed 4 November 2015).

Meier F, Elsweiler D and Wilson ML (2014) More than Liking and Bookmarking? Towards Understanding Twitter Favouriting Behaviour. In: ICWSM-14, Ann Arbor, MI. Available from: http://www.cs.nott.ac.uk/ pszmw/pubs/icwsm2014-favouriting.pdf (accessed 27 November 2015). 
Nagy P and Neff G (2015) Imagined Affordance: Reconstructing a Keyword for

Communication Theory. Social Media + Society 1(2). Available from:

http://sms.sagepub.com/content/1/2/2056305115603385 (accessed 9 October 2015).

Newton C (2015) Twitter officially kills off favorites and replaces them with likes. The Verge.

Available from: http://www.theverge.com/2015/11/3/9661180/twitter-vine-favorite-fav-

likes-hearts (accessed 3 November 2015).

Norman D (1990) The Design of Everyday Things. New York: Doubleday Business.

Norman DA (1988) The Psychology of Everyday Things. New York: Basic Books.

Norman DA (1999a) Affordance, conventions, and design. interactions 6(3): 38-43.

Norman DA (1999b) Affordance, conventions, and design. Interactions 6(3): 38-43.

Paßmann J, Boeschoten T and Schäfer MT (2014) The Gift of the Gab. Retweets cartels and gift economies on Twitter. In: Twitter and Society, New York, NY: Peter Lang, pp. 331344.

Postigo H (2016) The socio-technical architecture of digital labor: Converting play into YouTube money. New Media \& Society 18(2): 332-349.

Rochet J-C and Tirole J (2003) Platform Competition in Two-Sided Markets. Journal of the European Economic Association 1(4): 990-1029.

Schrock AR (2015) Communicative Affordances of Mobile Media: Portability, Availability, Locatability, and Multimediality. International Journal of Communication 9(0): 12291246.

Stanfill M (2014) The interface as discourse: The production of norms through web design. New Media \& Society: 1461444814520873. 
Stone B (2006) Six More Twitter Updates! Twitter Blogs. Available from:

https://blog.twitter.com/2006/six-more-twitter-updates (accessed 27 November 2015).

Styles K (2016) Twitter is 10 and it's still not a social network. The Next Web. Available from: http://thenextweb.com/opinion/2016/03/21/twitter-10-still-not-social-network/ (accessed 24 March 2016).

Sun H and Hart-Davidson WF (2014) Binding the material and the discursive with a relational approach of affordances. In: Proceedings of the 32nd annual ACM conference on Human factors in computing systems, ACM, pp. 3533-3542. Available from: http://dl.acm.org/citation.cfm?id=2557185 (accessed 20 January 2016).

Topolsky J (2016) The End of Twitter. The New Yorker. Available from: http://www.newyorker.com/tech/elements/the-end-of-twitter (accessed 24 March 2016).

Treem JW and Leonardi PM (2012) Social media use in organizations: Exploring the affordances of visibility, editability, persistence, and association. Communication yearbook 36: 143-189.

Tsukayama H (2016) The death of Twitter as we know it. The Washington Post, 11th February. Available from: https://www.washingtonpost.com/news/the-switch/wp/2016/02/11/thedeath-of-twitter-as-we-know-it/ (accessed 24 March 2016).

Twitter Help Center (n.d.) FAQs about tailored suggestions. Twitter Help Center. Available from: https://support.twitter.com/articles/20169941 (accessed 15 March 2016).

Van Dijck J (2013) The Culture of Connectivity: A Critical History of Social Media. New York: Oxford University Press.

Van Dijck J and Poell T (2013) Understanding Social Media Logic. Media and Communication 1(1): 2-14. 
Wellman B (2001) Physical place and cyberplace: The rise of personalized networking.

International journal of urban and regional research 25(2): 227-252.

Wellman B, Quan-Haase A, Boase J, et al. (2003) The Social Affordances of the Internet for Networked Individualism. Journal of Computer-Mediated Communication 8(3).

Available from: http://onlinelibrary.wiley.com/doi/10.1111/j.1083-

6101.2003.tb00216.x/abstract (accessed 20 January 2016).

Weltevrede E (2016) Repurposing digital methods: The research affordances of platforms and engines. Ph.D., Amsterdam: University of Amsterdam. Available from: https://wiki.digitalmethods.net/Dmi/RepurposingDigitalMethods (accessed 22 January 2016).

You H and Chen K (2007) Applications of affordance and semantics in product design. Design Studies 28(1): 23-38.

Zheng Y and Yu A (2016) Affordances of social media in collective action: the case of Free Lunch for Children in China. Information Systems Journal 26(3): 289-313.

\section{Endnotes}

\footnotetext{
${ }^{1}$ https://twitter.com/twocitylife/status/661701885498294272

${ }^{2}$ https://twitter.com/kumailn/status/661578296161800192

${ }^{3}$ https://twitter.com/ForresterRobert/status/661568358651924480

${ }^{4}$ https://twitter.com/deray/status/661597767052214272

${ }^{5}$ https://twitter.com/room34/status/661627844422864896

${ }^{6} \mathrm{https}: / /$ twitter.com/peterseibel/status/661571212061990913

${ }^{7}$ https://twitter.com/Longratter/status/661845126583590912
} 
${ }^{8}$ https://twitter.com/ultrabrilliant/status/661565224496009218

${ }^{9}$ https://twitter.com/aisyahzaili/status/661637978360418304

${ }^{10}$ We are not the first to propose a more complex understanding of the term. Gaver (1991) proposed notions of sequential and nested affordances, while McVeigh-Schultz and Baym (2015) suggest that affordances exist at various levels of abstraction, including: infrastructure, platforms and features.

${ }^{11} \mathrm{https} / / /$ developers.facebook.com/docs/marketing-apis

${ }^{12} \mathrm{https}: / /$ twitter.com/jack/status/20

${ }^{13} \mathrm{http}: / /$ knowyourmeme.com/memes/riptwitter

${ }^{14}$ See Weltevrede (2016) on the notion of the 'research affordances' of social media platforms as enabled by APIs.

${ }^{15}$ Twitter offers multiple APIs. Developers and researchers can employ the REST APIs for reading and writing Twitter data, and the Streaming APIs for accessing continuously updating or streaming Twitter data. Advertisers can employ the Ads APIs for integrating with Twitter advertising. See: https://dev.twitter.com/overview/documentation.

${ }^{16}$ However, as of March 2011 Twitter started limiting the kind of third-party applications that could be built using its APIs, which has halted the growth of its rich developer ecosystem (Bucher, 2013). In October 2015, Twitter's CEO Jack Dorsey officially apologized to thirdparty developers and promised to improve its relationship with them (Lunden and Olanoff, 2015).

\footnotetext{
${ }^{17} \mathrm{https}: / / \mathrm{dev}$. twitter.com/ads/overview

${ }^{18} \mathrm{https}: / /$ partners.twitter.com/

${ }^{19} \mathrm{https} / / /$ business.twitter.com/help/how-twitter-ads-work

${ }^{20} \mathrm{https} / / /$ blog.twitter.com/2016/resilient-ad-serving-at-twitter-scale

${ }^{21} \mathrm{http} / / /$ blog.instagram.com/post/141107034797/160315-news

${ }^{22}$ https://research.facebook.com/machinelearningarea/
} 
${ }^{23}$ https://support.twitter.com/articles/20169421

\section{Authors}

Taina Bucher, University of Copenhagen, wfg568@hum.ku.dk

Anne Helmond, University of Amsterdam, a.helmond@uva.nl 